\title{
The Full Calculate Of Discrete and Continuous Radiation of Hydrogen Plasmas for Solar Active Formations Based On The Equation System of Statistical Equilibrium
}

\author{
OSTAPENKO V. A. \\ Acad.Of Uas, Kiev, Ukraine; E-Mail: Osdar@Online.Ua
}

\begin{abstract}
The common calculations of discrete emission (in spectral lines) of hydrogen atoms and the continuous emission of hydrogen negative ions are made. The equation system (UST) of statistical equilibrium has been resolved in most full manner for radiation of hydrogen plasma in solar active formations. As initial data we use the observed intensities of spectral lines of hydrogen plasma. We use also all complex of modern information on solar flares and other flare-like formations and events. In the first time, the full system of equation of statistical equilibrium for hydrogen plasma without limiting of number of quantum states has been composited. We found also whole set of probabilities of elementary processes. We have outlined the physics of solar flares and flare-like events In the complex with analysis of spectral lines profiles and diffusion of radiation in turbulent (in fact, convective plasma). We have discovered that the energetics of hydrogen negative ions is by managed and it may be recreating in the laboratory. There is physical energetics of photons. Namely this energy from Suns' photosphere is used for ecological creation and operation with the organics on the Earth by life' Nature. We obtained also the real possibilities of creation of hand-made sun for people in any conditions on the Earth and outside the Earth.
\end{abstract}

Key words: physic energy, hydrogen plasma, the Sun, managed hydrogen energetics, full equation system of statistical equilibrium, UST, Layman-alpha radiation,

\section{Introduction}

The four stages in the study of the hydrogen atom emission in space objects are historically distinguished. They are planetary nebulae $\left(1940^{\text {th }}\right.$, Menzel \& Pekeris, 1935$)$, solar chromosphere $\left(60^{\text {th }}\right.$, Sobolev, $1958,1961)$, solar flares $\left(80^{\text {th }}\right.$, Ostapenko, 1974, 1984), and current sheets $\left(2010^{\text {th }}\right.$, Ostapenko, 1997,2005$)$. The fourth stage is a compatible solution of the UST equations system for discrete and continuous radiation of hydrogen is described here $\left(10^{\text {th }}\right.$, Ostapenko, 2005, 2012).

By emission line profiles we may to calculate only certain physical parameters. The main results of these studies are summarized by some authors (de Feiter, 1966; Smith \& Smith, 1966; Svestka, 1976). Selfagreed data about the physical conditions of the flare plasma can be obtained only from the solution of the equation system of statistical equilibrium. The problem is sufficiently complex and requires the previous discussion a number of additional problems. Namely: 1) The getting problem of enough complete and reliable observed data; 2) The developing problem of the composing and solving methodic of the UST equations system; 3) The obtaining problem of self-agreed and homogeneous cross-sections of all excitation and ionization elementary processes of the hydrogen atom; 4) The investigation problem of adequate models of the object being studied.

Ground-based studies of solar processes are made with the creation time of first telescope. It was Galileo (at the end of 1610) who discovered dark spots on the Sun (fig.4a). They were observed by other scientists as well. So, Jesuit Christopher Sheiner believed that spots were small bodies rotating around the Sun. Galileo's statement that these spots are located directly on the Sun's surface contradicted Aristotle' concepts about absolute imperishability and invariability of celestial bodies. As a result of debate with Sheiner, Galileo and order of Jesuits became enemies. Ideas of the Bible relation to the astronomy came to be widely used at that time. Now very much telescopes and scientific equipment investigated space objects and processes in all wavelengths. In 2009 it was marked the $400^{\text {th }}$ anniversary of Galileo's first telescopic observations (02.04.1609).

The main goal of this work is the obtaining of self-agreed and homogeneous cross-sections of excitation and ionization elementary processes of the hydrogen atom and the decision this task. We will discussed also main results and consequences for their practical using in people life.

\section{Elementary Processes Of The Hydrogen Atom Excitation}

Excitation and ionization of hydrogen atoms by the electron impact is one of some important problem of plasma physics. The self-agreed set of the elementary processes coefficients can be plotted with up to $30 \%$ 
The Full Calculate Of Discrete And Continuous Radiation Of Hydrogen Plasmas For Solar Active..

accuracy (Ostapenko, 1974). These data allow composing and solving the complete UST system, for the first time without limitation of the quantum levels number. It possibility has provided with the idea of using the semi-empirical expressions for excitation and ionization cross-sections of hydrogen atoms by the electron impact (Sampson and Golden, 1970). The basis and justification for the withdrawal of these semi-empirical expressions have used the logical assumption about the smooth changing of cross-sections with the level number. These expressions have chosen so that they matched up with the isolated existing and well known cross-sections which are available. In first, there are experimental excitation cross-sections from the first level to the second level and the ionization of the first level. Secondly, there are the cross-sections which are obtained in approaching the two bodies' problem of the classical theory and not elastic atom collisions (Gryzinski, 1959). It is known, the methods of classical approaching give correct results with coming electron energies in several times exceeding the threshold energy of a transition. Thirdly, these are the quantum-mechanical calculations by Vainstein et al. (1963), in which the interaction the atomic and coming electrons takes account. These calculations lead to the best coincidence of the calculated and experimental data in the threshold areas (Presnyakov, 1964).

The final expressions for the coefficients of bulky and they are difficult to use in practical calculations. So, Ostapenko (1974) presented them in much more convenient forms. The probability of hydrogen ionization by electron impacts that is expressed to one atom in the ground state and one free electron gives by follows:

$C_{n c}=5.46510^{-11} \sqrt{T_{e}} B_{n}(x)$,

Where: $x=1.578910^{5} n^{-2} / T_{e} ; B_{n}(x)=D_{n}(x)\left\{Q_{1}(x)+3 H_{n}\left[Q_{2}(n, x)+Q_{3}(x)\right]\right\}$.

Here: $\quad D_{n}(x)=1.3069 n^{3}<g_{b n}>x ; \quad Q_{1}(x)=E_{l}(x)-\frac{1}{3}\left[e^{-x}-x E_{3}(x)\right]$;

$Q_{2}(x)=\frac{x}{r_{n}^{\prime}-1}\left[E_{3-} r_{n}^{\prime}(x)-E_{2}(x)\right]+\frac{2 x}{2-r_{n}^{\prime}}\left[E_{3-} r_{n}^{\prime}(x)-E_{1}(x)\right]+\frac{1}{3-r_{n}^{\prime}}\left[e^{-x}-x E_{3-} r_{n}^{\prime}(x)\right]$;

$Q_{3}(x)=0.076 x\left[E_{0.5}(x)-E_{3}(x)\right]$.

In this expression $\left\langle g_{b n}\right\rangle$ - an average constant value of the gaunt radiation factor for bound-free transitions. The constants $\left\langle g_{b n}\right\rangle, H_{n}, r_{n}^{\prime}$ - for the first ten levels are given in the table (Sampson, Golden, 1970). The Integra exponentive function $E_{p}(x)$ has the form:

$E_{p}(x)=\int_{1}^{\infty} u^{-p} \exp (-x u) d u$.

The values of this function for $\mathrm{x}<20$ are given in the tables. The excitation probabilities of hydrogen atoms by electron impacts that recorded in a similar form, expressed by the formula:

$C_{n m}=5.4496 \frac{f_{n m}}{x \sqrt{T_{e}^{3}}} R_{n m}(x)$,

Where:

$$
x=1.578910^{5}\left(n^{-2}-m^{-2}\right) T_{e}^{-1}
$$

For stepwise transitions: $R_{n n+1}(x)=\Phi(x)+H_{n}\left[Z_{n n+1}(x)+Y_{n n+1} E_{2}(x)\right]$ (4)

For other transitions: $R_{n m}(x)=E_{1}(x)+H_{n}\left[Z_{n m}(x)+Y_{n n+1}\left[(1+x) E_{2}(x)-x E_{1}(x)\right] .(4 a)\right.$

Here:

$$
Z_{n m}(x)=0.19 S_{n m}^{2.5} x E_{3}(x) ; Y_{n m}=S_{n m}^{r_{n}}+A_{n}\left(S_{n m}-1\right) ; S_{n m}=\frac{E_{n m}}{I_{n}},
$$

Where $I_{n}$ - is the level $n$ ionization energy; $E_{n m}$ - energy transition between levels of $n$ and $m$. Constants $A_{n}$ and $r_{n}$ for the first ten levels presented in tables (Sampson, Golden, 1970). Function $\Phi(x)$ is defined by the equation:

$$
\left.\Phi(x)=x \int_{1}^{\infty}\left(1-\frac{1}{u}\right) \ln (u) \exp (-x u)\right) d u
$$

The ionization probability of $C_{n c}$ and excitation of $C_{n m}$ are calculated by Ostapenko (1974) for temperatures from 5000 to $20000 \mathrm{~K}$. the integrals - exponentive function $E_{p}(x)$ is obtained by the numerical manner. Function $\Phi(x)$ for $\mathrm{x}<10$ is taken from the tables (Golden, Sampson, 1971), and for $\mathrm{x}>10$ is calculated by the formula (Ostapenko, 1974): $\quad \Phi(x)=-0.49 x-1.30 . \quad$ (6)

Comparisons of the Ostapenko (1974) calculated values of $C_{n c}$ and $C_{n m}$ confirm good compliances with the well-known data for individual quantum transitions (Sampson \& Golden, 1970). Coefficients of the first levels are may deviated from well-known data. For stepwise transitions are used the refined formulas. This provides the differences of calculated values only within the $30 \%$. 
Transitions under the electron impact action from lower levels $n$ to higher levels $m$, for which the existing conditions of local thermodynamic equilibrium, can be combined with transitions in continuum $C$ in some «effective» continuum $M$.

$C_{n M}=\sum_{m=M}^{\infty} C_{n m}+C_{n c}$.

In this case, the $M$ is by any enough high level, which is already in $L T E$ conditions. Here, the fact is used that both radiative and collusion cross-sections are passed through the threshold ionization of continuous manner, transforming at $m \rightarrow \infty$ the excitation cross-section into the ordinary ionization cross-section (Menzel $\&$ Pekeris, 1935). This allows selecting the following corrected expression for the ionization cross section to one coefficient of an «effective» ionization to take into account all transitions $m \geq M$. Thus, if a UST equation to introduce «effective» coefficients, we can do not to limit the number of levels in solving of UST equations system that is usually done. The «effective» ionization probability that is calculated of one atom in the original state and one free electron has the appearance (Ostapenko, 1974): $C_{n M}=5.465110^{-11} \frac{\sqrt{T_{e}}}{S_{n m}^{4}} \Gamma_{n}(x)$,

where

$$
\Gamma_{n}(x)=D_{n}(x)\left\{Q_{1}(x)+3 H_{n}\left[S_{n m}^{r_{n}^{\prime}} Q_{2}(n, x)+S_{n m}^{2.5} Q_{3}(x)\right]\right\} .
$$

The $x$ calculates of (3a) formula, in this $m$ replacing of $M$. Sampson $\&$ Golden (1970) selected border level $M=$ 10. The probabilities of «effective» ionization for $M=10$ and $M=15$ are calculated by Ostapenko (1974).

\section{Excitation And Ionization Of Hydrogen Atom By Radiation Field}

«Effective» coefficients for radiative processes are given in the work of Johnson (1972), where is also proposed of convenient expressions for the calculation of gaunt factor:

$g_{n m}=g_{0}(n)+\frac{g_{1}(n)}{S_{n m}}+\frac{g_{2}(n)}{S_{n m}^{2}}$,

Where $S_{n m}=1-n^{2} / m^{2}$ and values $g_{i}(n)$ for $n \geq 3$ levels are calculated using the relationship:

$g_{0}(n)=0.9935+\frac{0.2328}{n}-\frac{0.1296}{n^{2}}$;

$g_{1}(n)=-\frac{1}{n}\left\{0.6282-\frac{0.5598}{n}+\frac{0.5299}{n^{2}}\right\}$;

$g_{2}(n)=\frac{1}{n^{2}}\left\{0.3887-\frac{1.191}{n}+\frac{1.47}{n^{2}}\right\}$.

For the first two levels

$\begin{array}{llll}n & g_{0}(n) & g_{1}(n) & g_{2}(n) \\ 1 & 1.133 & -0.4059 & 0.07014 \\ 2 & 1.0785 & -0.2319 & 0.02947\end{array}$

The Gaunt factor $g_{n m}$ passes the threshold ionization by continuous manner, turning in the normal factor $g_{n c}$ to bound-free transitions. This allows it using to calculate the effective coefficients of radiative processes.

Frequency dependence of radiation-medium interaction cross-section can have resonances, which are caused by dissipation or absorption in lines. Optical thickness in a line is usually much higher than the optical thickness in continuous spectrum. Radiation spectrum that passed through such medium has absorption lines. Radiation spectrum of optically thin medium has emission lines. If line's optical thickness exceeds one, the intensity and radiation in the center of the line has $\sim B_{\omega}(T)$ and the line will be saturated. As optical thickness further increases, the intensity in the center of the line remains unchanged and its width increases. If optical dense medium has a temperature which drops closer to the surface, the radiation on spectral line frequencies will come out of a cooler part of the medium. In this case, the optical dense medium (photosphere) radiates of the absorption lines (Ambartsumjan et al., 1952). $I\left(x_{n}\right)=I\left(x_{m}\right) e^{-\tau_{a}\left(x_{m n}\right)}$

Full flux attenuation is determined by total optical thickness $\tau_{\alpha}=\Sigma \tau_{\alpha}$. A matter between points $\mathrm{x}_{m}$ and $\mathrm{x}_{n}$ is considered to be optically thin at $\tau_{a}\left(\mathrm{x}_{m n}\right)<<1$ and optically thick at $\tau_{a}\left(\mathrm{x}_{m n}\right)>1$. In general optical thickness depends on radiation frequency $\omega$. If any optically thin region generates its own thermal radiation, its intensity will equal (Conway, 1962):

$I(\omega)=B_{\omega}(T)\left[1-e^{-\tau_{a}\left(x_{m n}, \pi\right)}\right]$, 
where $B_{\omega}(T)$ - intensity of absolute black body emission. If optical thickness of radiation region equals $\tau \gg 1$, the properties of output radiation is determined by external layer with $\tau \sim 1$. In this case, $I(\omega) \sim B_{\omega}(T)$ with temperature $T$, which is peculiar for this layer.

Line profile of a homogeneous chromospheres' emission feature, which is located in field of photospheres' emission $I_{\lambda}^{\oplus}$ (Conway, 1962): $T\left(v, \tau_{n m}\right)=S\left[1-e^{-\tau_{n m} \alpha(v)}\right]+I_{p h} e^{-\tau_{n m} \alpha(v)}$,

Where $\alpha(\mathrm{v})=\mathrm{e}^{-\mathrm{v} v}$ is a profile of absorption coefficient for thermal movements of absorbing atoms. The selfabsorption factor estimates the number of escaped quants from optically dense medium.

$$
F\left(\tau_{n m}\right)=\frac{E\left(\tau_{n m}\right)}{E\left(\tau_{n m}=0\right)}=\frac{1}{\sqrt{\pi} \tau_{n m}} \int_{-\infty}^{\infty}\left[1-e^{-\tau_{n m} \alpha(v)}\right] d v .
$$

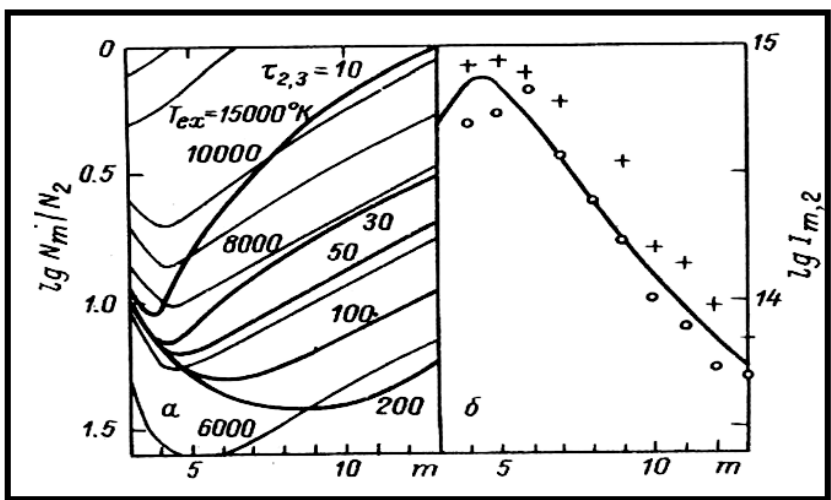

Fig.1. All experimental errors in the observable Balmer line intensities are eliminated using the LTE laws.

Dependences of level populations ratio $N_{m} / N_{2}$ from top-level number $m$ and the optical thickness of the object in the $H_{\alpha}$ line (left), Balmer decrement (right) line intensities for powerful flares $\left(T_{e x}=7200 \mathrm{~K}\right)$. Thin lines show $N_{m} / N_{2}$ that is found by the Boltzmann formula. Crosses and cycles showing the intensity of two powerful flares.

The optical thicks in lines of one spectral series (f. e., hydrogen Balmer series) are not independent. They are connected with each other by the ratio (g5) through the wavelength $\lambda_{n m}$ and oscillator force $f_{n m}$ (a number of possible electron states on the quantum level $n$ that allowed by rules of the quantum selection $\left(\sim 2 n^{2}\right)$ ). Optical thicknesses in lines of Balmer series are related to each other by the ratio (Eljashevich, 1962): $\tau_{2 m}=$ $\tau_{23} \frac{\lambda_{2 m} f_{2 m}}{\lambda_{23} f_{23}}$

Here $\lambda_{n m}$ is a wavelength for transition from level $m$ to level $n$, and $S_{n m}=1-n^{2} / m^{2}$. Oscillator forces $f_{n m}$ for transitions from $n$ to $m$ can be easily calculated by means of gaunt factor $g_{n m}$ :

$f_{n m}=1.96028 \frac{n g_{n m}}{m^{3} S_{n m}^{3}}$,

and probabilities of spontaneous transitions.

$A_{m n}=f_{n m} \frac{2\left(1-S_{n m}\right)}{3 \lambda_{n m}^{2}}$

Strengthening (weakening) of spectral line radiation is characterized by the residual intensity value $r_{v}$ that is the intensity ratio $I_{v}$ to the frequency $v$ inside the line $\left(r_{v}=I_{v} / I^{o}\right)$. The dependence of residual intensity $r_{v}$ on the frequency $v$ is called a spectral line profile. Full emission (absorption) flux in a unit solid angle on all inside the line frequencies is called full spectral line intensity. The measure that shows part of continuous spectrum is equivalent to full spectral line intensity is called equivalent width $W_{v}$ of a spectral line.

Probabilities of photo ionization are calculated of the formula (Johnson, 1972):

$A_{M n}=5.19710^{-14} \sqrt{x_{n c}^{3}} \exp \left(x_{n c}\right) \sum_{i=0}^{2} \frac{g_{i}(n)}{S_{n m}^{i}} E_{i+l}\left(S_{n M}, x_{n M}\right)$, 
$X_{n c}=\frac{h v_{n c}}{k T_{e}}=\frac{1.578910^{5}}{n^{2} T_{e}} \frac{1.578910^{5}}{n^{2} T_{e}}$.

The coefficient photo ionization, under the influence of the solar (outer) radiation, taking into account stimulated emission is calculated of the formula (Ostapenko \& Kurochka, 1978):

$B_{n M} J_{n M}^{\bar{\oplus}}=\frac{7.8710^{9}}{n^{5}} \int_{S_{n M} x_{n c}}^{\infty} g_{n M} \frac{e^{-\alpha x}}{x} \frac{1-e^{-x}}{1-e^{-\alpha x}} d x$,

Where: $\alpha=T_{n c} / T_{\odot}$. When the given level populations $N_{m}$ and temperature $T_{\odot}$, the temperature of $T_{n c}$ in the object that is being studied, is determined by the ratio of

$-\frac{h v_{n c}}{k T_{n c}}=\ln \left(\frac{n^{2}}{100} \frac{N_{10}}{N_{n}}\right)-\frac{h v_{n c}}{k T_{e}}$.

The temperature of the solar radiation $T_{\odot}$ can be found according to Dupree et al (1973). Convenient to use approximate expression (Ostapenko, Rossada, 1976):

$T^{\oplus}=5000 \exp \left\{0.184 \cos \left[\frac{\pi}{2} \operatorname{abs}(\lg \lambda)\right]\right\}$.

Formula (13) found on the Makarova \& Kharitonov (1968) data and describes the experimental results from Balmer series frontier (3680 ̊́) to $0.1 \mathrm{~cm}$.

If the Gaunt factor is known, it is easy to calculate the exact value of the oscillators force $f_{n m}$ (expression g6) and the probability of spontaneous transitions $A_{m n}$ (expression g7). The calculation problems of the radiation energy $E_{m n}$ in the lines are related to the difficulty of taking into account of the probability of quanta escape $\beta_{n m}$ from the environment (Sobolev, 1959; Ostapenko, 1974). The probability of quanta escape $\beta_{n m}$, averaged across the optical thickness of the object is equal to the determining of the factor self-absorption $F\left(\tau_{n m}\right)$ (formulae g4 of entered Conway (1962)). The excess number of transitions $m \rightarrow n$ above backward transitions, which occurs under the action of its own field of radiation, will be $N_{m} A_{m n} \beta_{n m} \equiv N_{m} A_{m n} F\left(\tau_{n m}\right)$, (14)

\section{The Lyman-Alpha Radiation Of Hydrogen Plasmas}

The Lyman series is the series of energies required to excite an electron in hydrogen from its lowest energy state to a higher energy state. The case of particular interest for cosmology is where a hydrogen atom with its electron in the lowest energy configuration gets hit by a photon and is boosted to the next lowest energy level. The energy levels are given by $E_{n}=-13.6 \mathrm{eV} / \mathrm{n}^{2}$ and the energy difference between the lowest $(\mathrm{n}=1)$ and second lowest $(\mathrm{n}=2)$ levels corresponds to a photon with wavelength $1216 \AA$. The reverse process can and does occur as well, where an electron goes from the higher $n=2$ energy state to the ground state, releasing a photon of the same energy.

The temperature of Lyman-alpha radiation $T_{21}$ is important to know when physical conditions in the emission formations are found by the solution method of UST equations system of the statistical equilibrium. This is the problem of assessing the one of main parameters of the model, which is the plasma density, which do not found by observations from the Earth' surface.. When the temperature is $<8000 \mathrm{~K}$ the majority of hydrogen atoms is located in the ground quantum state. All transitions on the first level are accompanied by radiation in the $U V$ region. At the same time, the relationship between the electron temperature $T_{e}$ and temperature $T_{21}$ is unknown. Any researcher must to consider of the $T_{21}$ as a free parameter (Sobolev, 1961). We may to use the two extremely cases. The $A$ case when optical thicknesses is zero in the Lyman series; Secondly, this is the $B$ case: the LTE conditions are in the Lyman lines (Baker \& Menzel, 1938). We believe physical parameters by homogeneous and without change along the line of sight. In calculations used the hydrogen atom model with the levels number $m=9$ and continuum. The hydrogen density:

$n_{H}=n_{e}+\sum_{m=1}^{\infty} n_{m}$,

The source parameters are $n_{H}, T_{e}$ and $l$ (the length of the object along the line of sight), as well as the Doppler width $\Delta \lambda_{D} / \lambda=0.710^{-4}$. The $T_{21}$ may be found from the stationary equation for first level: 


$$
\frac{N_{2}}{N_{1}}=\frac{n_{e} C_{1 c}+n_{e} \sum_{m=2}^{M} C_{1 m}+B_{1 c} J_{1 c}^{\oplus}+\sum_{m=2}^{M} B_{1 m} J_{1 m}^{\oplus}}{n_{e} \sum_{m=2}^{M} \frac{N_{m}}{N_{2}} C_{m 1}+\sum_{m=2}^{M} \frac{N_{m}}{N_{2}} A_{m 1} \beta_{1 m}+\frac{n_{e}^{2} l}{N_{2}}\left[A_{c 1} \beta_{1 c}+n_{e} C_{c 1}\right]} .
$$

Here $C_{I m}$ and $C_{I c}$ are the excitation and ionization coefficients of the hydrogen atoms by electron impacts. Numerical values of the coefficients are given in Ostapenko (1974). The $A_{m l}$ and $B_{l m}$ are the Einstein ratios for radiative transitions. The number of transitions in the result of the excitation by external radiation field is calculated according to the formula (Chultem \& Yakovkin, 1974)

$N_{l} B_{l m} J_{1 m}^{\oplus}=I_{1 m}^{\oplus} W \beta_{l \mu} \tau_{1 \mu} \mu_{l \mu}$,

$\mu_{1 \mu}=0.11210^{18} \frac{\Delta \lambda_{D}}{\lambda} \lambda^{2}$,

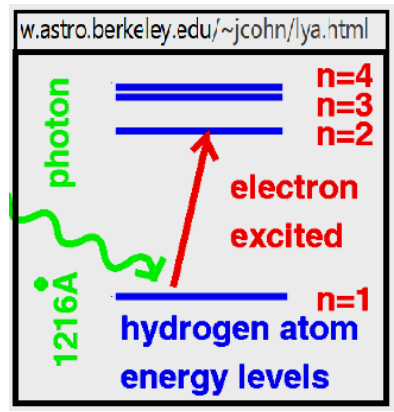

and $W$ - dilution factor. To calculate the probability of outlet photons used

approximate expression. The $N_{l} B_{l c} J_{1 c}^{\oplus}$ coefficient is presented in the same form as (17). So, the amount of solar energy that is absorbed in the continuum can be written:

$E_{a b s}=\int_{\lambda_{0}}^{0} I_{\lambda}\left(1-e^{-\tau_{\lambda}}\right) d \lambda=I_{v_{0}}^{\oplus} \frac{\exp \left(\frac{h v_{0}}{k T^{\oplus}}\right)}{v_{0}^{3}} \int_{v_{0}}^{\infty}\left(1-e^{-\tau_{v}}\right) v^{3} \exp \left[-\frac{h v}{k T^{\oplus}}\right] d v$.

Using the expression for the quanta outlet for continuum, we get expression for photons number:

$N_{l} B_{l c} J_{1 c}^{\oplus}=\frac{E_{a b s}}{h v_{1 c}}=0.29710^{11} I_{\lambda_{0}}^{\oplus} \lambda_{0}^{2} \tau_{l c} \mu_{l c}=\mu_{l c} I_{\lambda_{0}}^{\oplus} \tau_{l c} \beta_{l c}$.

Photon energy $h v_{I c}$ is taken in some average value for $\lambda=850 \mathrm{~A}$. The number of escape photons in the Lyman continuum, with the required here precision, appears in the form:

$n_{e}^{2} l A_{c l} J_{l c}=\frac{E_{r a d}}{h \bar{v}_{1 c}}=0.12310^{-11} \frac{n_{e}^{2} l}{\sqrt{T_{e}}} \beta_{l c}$.

The population of the second level of the any flare powers remains the same (Krivsky \& Kurochka, 1974). The populations of the upper levels are little influenced on the results of the solution of the equation for the first level equilibrium.

To simplify of calculations, the ratios $N_{m} / N_{2}$ of population levels are described by the Boltzmann formula with the $T_{e x}=6000 \mathrm{~K}$ temperature (Kurochka et al., 1975). To fainter emission formations $\left(T_{e x}<6000 \mathrm{~K}\right)$, level populations above the third virtually no effect on the $N_{2} / N_{l}$. The $n_{e}$ is found from the equation of ionization equilibrium

$n_{e} \sum_{m=1}^{M} N_{m} C_{m c}+\sum_{m=1}^{M} N_{m} B_{m c} J_{m c}^{\oplus} W=$
$=n_{e}^{2} l\left[A_{c l} J_{l c}+\sum_{m=2}^{M} A_{c m}+n_{e} \sum_{m=1}^{M} C_{c m}\right]$,

As well as of equations $\frac{n_{e}^{2}}{n_{2}}=\frac{2\left(2 \pi m_{e} k T_{e}\right)^{3 / 2}}{g_{2} h^{3}} \exp \left[-\frac{h v_{2 c}}{k T_{2 c}}\right]$,

$n_{2}=4 n_{1} \exp \left[-\frac{h v_{12}}{k T_{21}}\right], \quad n_{H}=n_{e}+n_{1}$.

If solve the system of equations (22) against $n_{e}$, we get

$n_{e}=\frac{\mathrm{X}(T)}{2}\left[\sqrt{\left(1+\frac{4 n_{H}}{\mathrm{X}(T)}\right)}-1\right], \quad \mathrm{X}(T)=\frac{\left(2 \pi m_{e} k T_{e}\right)^{3 / 2}}{h^{3} \exp \left[\frac{h v_{12}}{k T_{21}}+\frac{h v_{2 c}}{k T_{c 2}}\right]}$. 
The $n_{e}$ values are being found of the (21) \& (23) equations by the sequential approximation method. The $n_{e}$ first value is obtained in $T_{e}=T_{21}$ supposition as well as in supposing that the hydrogen ionization is caused by Balmer Sun's continuum (with $T_{2 c}=5600 \mathrm{~K}$ ). Substituting the obtained $n_{e}$ in the (16) equation, we find the first approximation of $N_{2} / N_{l}$. The new $T_{21}$ approach is used to obtain a new $n_{e}$ approach. The $T_{21}$ data are found for set of $n_{H}, T_{e}$ and $l$ (Ostapenko, 2012). Their analysis shows that for the actual values of the $10^{10}<n_{H}<10^{14} \mathrm{~cm}^{-3}$ by hydrogen density and the $10^{5}<l<10^{9}$ its facility along the beam, the $T_{21}$ may be $7300<T_{21}<T_{e}$. The minimal value $T_{21}=7300 \mathrm{~K}$ is determined by the solar Lyman-alpha radiation of $T_{21}=7600 \mathrm{~K}$. Note that amongst the chromosphere formations was not such situations, where condition $T_{e}=T_{21}$ would is installed.

The LTE conditions (case $B$ ) are only achieved in the transition zone at the $n_{H}>10^{14} \mathrm{~cm}^{-3}$ near the photosphere where the Fraunhofer lines are formed. The case $A$, when optical thicknesses in the Lyman-alpha line are $n_{H}<10^{10} \mathrm{~cm}^{-3}$, the solar atmosphere becomes by the corona.

Later, in the full UST equations system is conveniently used the (24) approximate expression, which has replaced the equation for the first level:

$$
\begin{aligned}
& T_{2 l}=7300+\frac{T_{e}-7300}{1+e^{-x}}, \\
& x=\lg n_{e}^{2} l-29.4-3.14\left(\lg T_{e}-3.7\right) .
\end{aligned}
$$

The data shows that in chromosphere formations the local LTE is always absent. The obtained results for the first time have given a quantitative definition of the different (physically reason now) zones of the Sun's atmosphere. So, the transition zone is begun after plasma density achieving of $n_{H} \sim 10^{14} \mathrm{~cm}^{-3}$. Here the photosphere absorption metal lines are formed and the local LTE as the case $A$ of the Menzel approximation is achieved in Lyman-alpha hydrogen line (Baker, Menzel, 1938). The photosphere actually begins by achieving the $n_{H} \sim 10^{18} \mathrm{~cm}^{-3}$ plasma density. The hydrogen plasma is not transparent in the continuous spectrum during the negative hydrogen ion excitation (fig.2).

Observations of Lyman-alpha radiation in the case of small densities of hydrogen are given in fig.3. Dubbed a Lyman-alpha blob, an enormous cloud of hydrogen gas spans several hundred thousand light-years, a composite of $x$-ray, optical, and infrared data from space and ground based observatories. The gigantic, amoeba-like structure is seen as it was when the universe was a mere 2 billion years old ( 12 billion years ago). Lyman-alpha blobs are so called because they strongly emit radiation due to the Lyman-alpha emission line of hydrogen gas. Normally, Lyman-alpha emission is in the ultraviolet part of the spectrum, but the blobs are so distant, their light is redshifted to (longer) optical wavelengths. X-ray data (blue) indicates the presence of a supermassive black hole feeding at the center of an active galaxy embedded in the blob. Radiation and outflows from the active galaxy are thought to be a source for energizing and heating the blob's hydrogen gas. In fact, Lyman-alpha blobs could represent an early phase in galaxy formation.
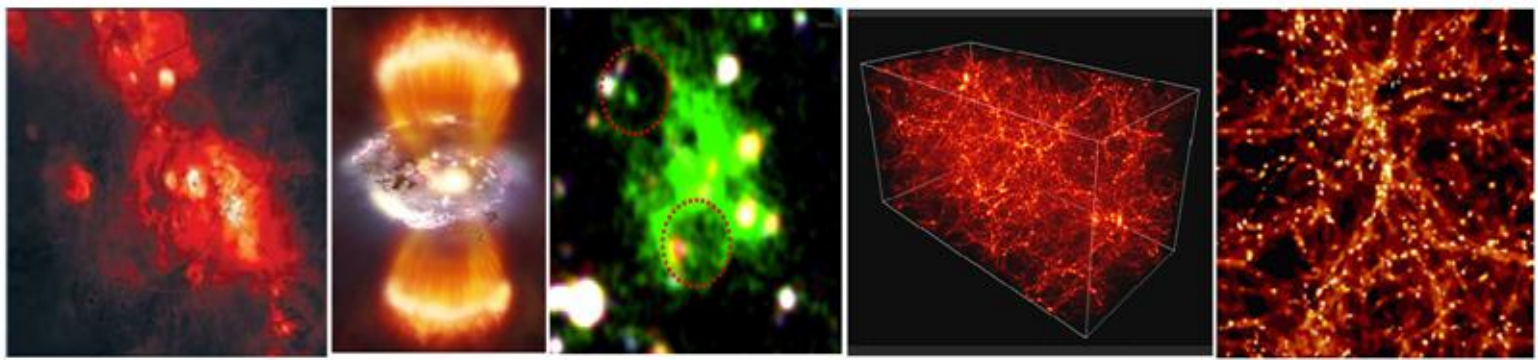

Fig.2. Lyman-alpha blobs are some of the largest individual objects in the observable Universe as the Menzel approximation case $A$ of transparent plasma for Lyman-alpha radiation $\left(\mathrm{n}_{\mathrm{H}}<<10^{10} \mathrm{~cm}^{-3}\right)$.

$\boldsymbol{a}$. The near black hole areas in the Lyman-alpha light that is formed by falling gas in black hole gravitational field and it traces the dark matter. http://w.astro.berkeley.edu/ jcohn/lya.html

$\boldsymbol{b}$, $\boldsymbol{c}$. Layman-alpha blob (APOD-02.07.2009). Some of these gaseous structures are more than 400,000 lightyears across. The gigantic, amoeba-like structure is seen as it was when the universe was a mere 2 billion years old (about 12 billion years ago). X-ray data (blue) indicates the presence of a super massive black hole feeding at the centre of an active galaxy embedded in the blob.

$\boldsymbol{d}$, $\boldsymbol{e}$. The 3D map of clusters of galaxies in hydrogen light as the dark matter distribution. http://pics-aboutspace.com/dark-matter-map-of-the-universe? $\mathrm{p}=1$ 


\section{The UST Equations System Without Limiting Of The Level Number}

The equations system of statistical equilibrium allows to obtain in addition to the basic parameters of the object $n_{H}, T_{e}$ and $l$ also optical thicknesses in spectral lines. As the initial data the observed equivalent widths of the Balmer series lines of solar flares are used. The photosphere radiation is believed as external radiation field. We introduced also the concept of structural heterogeneity of plasma that consists of separate elements of macro turbulence. Observed populations of levels $N_{m}^{\prime}(m>3)$ are obtained to amplify on a flare porosity Q: $N_{m}=N_{m}^{\prime} Q$. The $\mathrm{Q}$ is the ratio of visible flare square to a square occupied by these elements (Kurochka et al., 1975):

$Q=S_{\text {obs }} / \sum_{i} S_{i}$.

Thus, the porosity is entered into the UST system through level populations $N_{m}$ and it can be found at a divergence system minimum of equations. The main differences between our method and such type methods are next. (a) The specific physical conditions in solar flares by various powers are of the identifying task. (b) For the first time the real expression (24) for the Layman radiation field are using in the UST equations system. (c) The complete, without limiting the levels number, the UST equations system (7) is solving due to the introduction of «effective» continuum. (d) All elementary processes in plasma and most new data about them take into account. (e) The optical thicknesses of the lines are calculated simultaneously with the main physical parameters of $n_{H}, T_{e}$ and $l$. (f) The porosity flare problem is analyzed. We put the challenge to find physical data, just the ones specific to flares given power, which is provided by the observed emission in hydrogen lines.

We have assumed of pure thermal excitation mechanism of the flares in the model of compacted plasma layer, which is located horizontally in the chromosphere in the photosphere radiation field. All physical parameters in the layer are considered to be uniformly distributed along the line of sight. Although flares are changing sufficiently quickly, we can assume that the stationary conditions are well executed for each level at any point of time (Yakovkin, 1963):

$$
\begin{aligned}
& n_{e} \sum_{j=1}^{n-1} N_{j} C_{j n}+\sum_{j=1}^{n-1} N_{j} B_{j n} J_{j n}^{\oplus}+n_{e} \sum_{m=n) 1}^{M-1} N_{m} C_{m n}+\sum_{m=n+1}^{M-1} N_{m} A_{m n}+ \\
& +\sum_{j=1}^{n-1} N_{n} A_{n j}\left(1-\beta_{j n}\right)+n_{e}^{2} l A_{M n}+n_{e}^{2} l C_{M n}= \\
& =n_{e} \sum_{j=1}^{n-1} N_{n} C_{n j}+n_{e} \sum_{m=n+1}^{M-1} N_{n} C_{n m}+\sum_{j=1}^{n-1} N_{n} A_{n j}+\sum_{m=n+1}^{M-1} N_{m} A_{m n}\left(1-\beta_{n m}\right)+ \\
& +\sum_{m=n+1}^{M-1} N_{n} B_{n m} J_{n m}^{\oplus}+n_{e} N_{n} C_{n M}+N_{n} B_{n M} J_{n M}^{\oplus} W .
\end{aligned}
$$

We introducing the notations:

$$
\begin{aligned}
& A_{I}(n)=\sum_{m=n+1}^{M-1} N_{m} A_{m n} \beta_{n m}-\sum_{j=1}^{n-1} N_{n} A_{n j} \beta_{j n} ; \\
& A_{2}(n)=\sum_{j=1}^{n-1} N_{j} B_{j n} J_{j n}^{\oplus}-\sum_{m=n+1}^{M-1} N_{n} B_{n m} J_{n m}^{\oplus}-N_{n} B_{n M} J_{n M}^{\oplus} W ; \\
& \frac{A_{3}(n)}{n_{e}}=A_{3}^{\prime}(n)=\sum_{j=1}^{n-1}\left(N_{j} C_{j n}-N_{n} C_{n j}\right)-\sum_{m=n+1}^{M-1}\left(N_{n} C_{n m}-N_{m} C_{m n}\right)-N_{n} C_{n M} .
\end{aligned}
$$

The equation of the ionization equilibrium has the form:

$$
\begin{aligned}
& n_{e}^{3} l \sum_{n=1}^{M-1} C_{M n}-n_{e} \sum_{n=1}^{M-1} N_{n} C_{n M}+n_{e}^{2} l\left\{A_{M l} \beta_{I M}+\sum_{n=2}^{M-1} A_{M n}\right\}- \\
& -W \sum_{n=1}^{M-1} N_{n} B_{n M} J_{n M}^{\oplus}=0 .
\end{aligned}
$$

The complete UST system of equations consists of the stationary equation for the first level in the form of (24), the eight equations for each level (26) and the equation of the ionization equilibrium (28). The effective continuum we believe the $\mathrm{M}=10$ as all higher levels ( $\mathrm{m}>10$ ) are already considered in the LTE (Kingston, 
1964). As solution we may to search the set of dependencies $\left(n_{e}^{2} l\right)_{m}=f\left(n_{e}\right)$ foe set of $T_{e} \& \tau_{23}$ values. Of expressions (27), for all levels of $\mathrm{m}<\mathrm{M}$, we have:

$$
n_{e}^{2} l=\frac{-n_{e} A_{3}(n)-A_{1}(n)-A_{2}(n)}{n_{e} C_{M n}+A_{M n}} .
$$

This is especially useful when working with computers, in terms of output to monitor the results of the current computation in axis: $n_{e}^{2} l, n_{e}$ (fig.1). At small values of $n_{e}$ :

$n_{e}^{2} l=\frac{-A_{1}(n)-A_{2}(n)}{A_{M n}}=$ const.

Curves go parallel axis $\operatorname{lgn}_{e}$, characterized by the fact that in the excitation of a given level involved the radiative processes only. With increasing $n_{e}$ in the level balance begin to manifest electron impacts, the curves are drawn together, intersect and diverge again. When enough large values of $n_{e}$ (Polupan \& Yakovkin, 1965), radiative processes are becoming negligible against electron impacts and dependence of $n_{e}$ in (29) disappears again. Then:

$n_{e}^{2} l=\frac{-A_{3}(n)}{C_{M n}}=$ const.

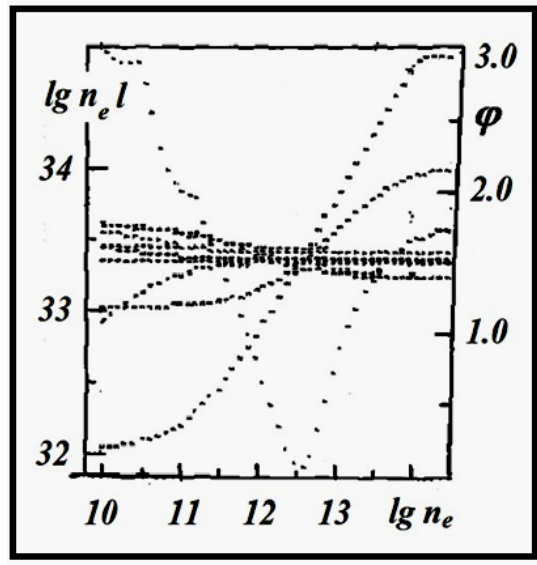

Fig.3. The solution method of the $\boldsymbol{U} \boldsymbol{S T}$ equation statistical equilibrium system for the linear emission for the flare of the middle power. The dependencies $n_{e}^{2} l\left(n_{e}\right)$ for $\Theta=1, T_{e}=9500 \mathrm{~K}$, and $\tau_{23}=74$ on monitor screen (Ostapenko \& Kurochka, 1978).

The lines are again becoming a parallel of axis $l g n_{e}$, pointing out that now in the excitation levels prevail of electron processes. The progress curves near the crossing point allowed judging the relative contribution of various processes in the excitation of each level. With the right choice of model using object and initial parameters $-n_{H}, T_{e}, l, \tau_{23}$, and $\Theta$, as well as in the absence of other processes, the curves for all levels must converge in a single point, which determines the values of the $n_{e}$ and $n_{e}^{2} l$. In practice, the perfect intersection of curves is not achieved. The quantitative criterion of levels discrepancy is derived from the

introduction of the relative «discrepancies» $\varphi: \quad \varphi=\sum_{n=2}^{M} \frac{\left[\sum_{i=1}^{5} A_{i}(n)\right]^{2}}{\left[A_{i}(\max )\right]^{2}}$,

Where, in the previous (27) notation introduced in addition:

$A_{3}(n)=n_{e} A_{3}^{\prime}(n) ; A_{4}(n)=n_{e}^{3} l C_{M n} ; A_{5}(n)=n_{e}^{2} l A_{M n}$.

We choose relative terms as absolute $A_{i}(n)$ means are significantly different values for each level.

The UST solving example is considered for medium power flares (ball $1 n$; Kurochka \& Ostapenko, 1978) with parameters $-\tau_{23}^{\prime}=100, T_{e x}=5800 K i \frac{\Delta \lambda_{D}}{\lambda}=0.710^{-4}$. By specifying different values for $T_{e}$, and $\tau_{23}$, we building the $\varphi=\varphi\left(n_{e}\right)$ dependences and find the appropriate solution of the UST equations system (type of fig.2). Each similar decision used to build dependencies $\varphi_{\min }=\varphi_{1}=\varphi_{\min }\left(\tau_{23}\right)$, as well as $\varphi_{\min }=\varphi_{2}=\varphi_{\min }\left(T_{e}\right)$ 
(fig.2). These curves allow to confidently assessing the optimal values for $T_{e}, \tau_{23}$ (with a fixed value of porosity $\Theta)$. The curve $\varphi_{\min }=\varphi_{3}=\varphi_{\min }(\Theta)$ shows that the porosity in flares is absent. This is an unexpected result, since the observing area of any flare and the calculated length of the flare along the beam of the different orders. The final solution is $n_{e}=510^{12} \mathrm{~cm}^{-3}, T_{e},=9500 \mathrm{~K}, l=110^{7} \mathrm{~cm}, \tau_{23}=74, \Theta=1,\left(n_{e}^{2} l=2.610^{32} \mathrm{~cm}^{-5}\right)$. Because, the observing flare size $\sim 10^{9} \mathrm{~cm}$ and the calculating one $\sim 10^{7} \mathrm{~cm}$, the existence of the fine flare structure was settled to onset an idea of a cluster of moving compacted emission elements. At first glance, the analysis of profiles of spectral lines (Ostapenko, 2012; Chapter 2) has also confirms this. The porosity lack $(\Theta=1)$ creates a framework to search for a suitable flare model. It is difficult to reconcile the model flares as a horizontal compacted plasma layer with the conglomerate of moving emission elements. Having small beam lengths, they photosphere screens.

When solving the UST equations system we used assumptions that the tenth level is already in a detailed LTE state $(M=10)$. To the fidelity test of such assumptions the same $U S T$ equations system when $M=15$ has solved. Calculations have shown that the increase of «effective» the continuum to the 15th level of the results do not change appreciably. Because the approximation of $M=10$ can be considered fully satisfactory. The fallout of the fourth level of the overall picture of dependencies $n_{e}^{2} l\left(n_{e}\right)$ on fig. 1 may be an influence effect of the levels «over excitation» by external radiation field in the photosphere of the growing influence of electron impact processes. The behavior of all the higher levels have obeys by the LTE (Ostapenko, 2012; formula 4.5.9).

We analyzed detail (Ostapenko, 2011; 2012, table 4.5.1) the energy balance is given for transitions between the quanta levels for the flares of all power. The total energy of flares is provided by own thermal excitation mechanism (90\%) with regard to the fields of Sun' radiation (10\%). Most energy (60\%) radiates in the Balmer series. The Balmer continuum, which is usually not stand out against the photosphere background, emitted to $45 \%$, and in the intensities line $H_{\alpha}$ in flares is $20 \%$ of the whole energy of the Balmer series. Central intensity of the $H_{\alpha}$ line:

$$
I_{32}=\frac{0.56810^{8}}{4 \pi \Delta \lambda_{D} 2 v_{1 / 2}}=2.210^{6} \frac{\operatorname{erg}}{\mathrm{cm}^{2} \operatorname{cster} A}\left(\frac{I_{32}}{I_{\lambda}^{\oplus}}=0.70\right) .
$$

Then, as the boundaries of the Balmer continuum:

$I_{c 2}=4.310^{-23} \frac{n_{e}^{2} l}{T_{e}^{3 / 2}}=1.210^{4} \frac{\mathrm{erg}}{\mathrm{cm}^{2} \operatorname{csterA}}\left(\frac{I_{c 2}}{I_{\lambda}^{\oplus}}=0.004\right)$.

The analysis calculated for solar flares shows that the contribution of the external radiation in the levels population of $m>4$ is essential. The fourth level is appeared to be a transitional and a «overpopulation» next to it levels, until a detailed energy balance is not installed due to electron impacts, leads to unbalanced of the fourth level (fig.3).
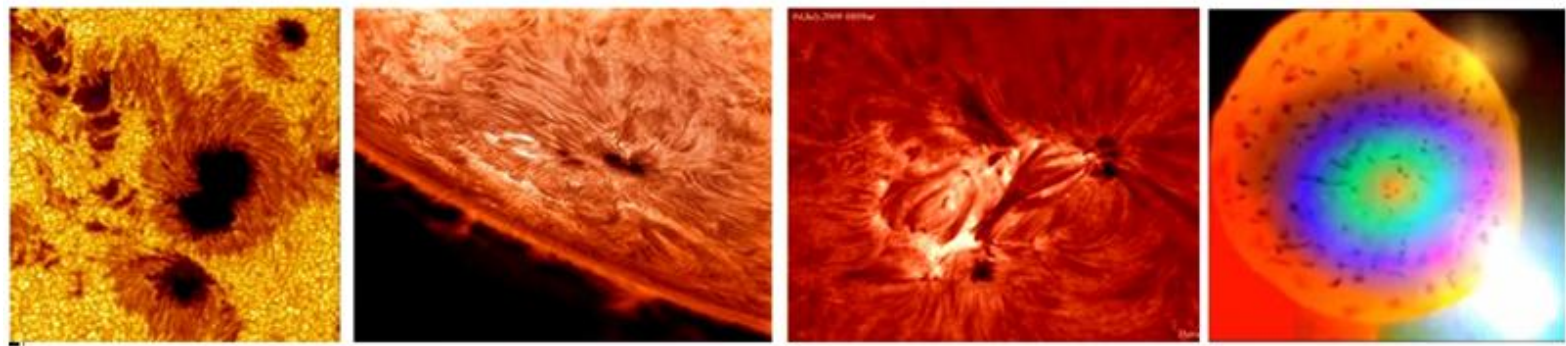

Fig.4. Solar spots and flares in a white light (photosphere) and in $\mathrm{H}_{\alpha}$ line (chromosphere).

a. A group of sunspots in a white light

http://www.ifdawn.com/esa/ sune.htm

b. One of the biggest sunspots in $24^{\text {th }}$ solar cycle.

http://wppc.conforums.com/ index. cgi. board $=$ World\&num $=$

c. Solar chromosphere' flare of B-class. http://www.greatdreams.com/ solar/2009/space-weather-july-2009.htm

d. $C M E-1$ [jet (white color), shock wave] of a huge flare 02.08.2010 and CME -2 (red to the left) - tsunami on photosphere. www.youtube.com/watch?v= MPXBIYSYCUQ\&feature=related

\section{The Thermal Excitation Mechanism And The «Physical Conditions» In Flares}


Physical characteristics of 50 real and average flares are calculated in all their energy range (Ostapenko, Polupan, 1984). As the flare model selected a horizontal layer of chromosphere plasma. In this model all principal conclusions about the physical conditions of the flare plasma are made. But already by the end of 1970's many representations about chromosphere flares go into explicit contradiction with the data in the microwave, UV, and X-ray ranges as well as with theoretical insights about possible mechanisms of flares. It brings in the first place the task of comparison physical, dynamic and spatial flare data. Conditions for success are the choice of an adequate flare model in the optical range

With the purpose of further analysis of the model the physical conditions are calculated in the whole range of flare powers. The flare model is static horizontal layer of pure hydrogen plasma in chromosphere. The object is believed to be homogeneous, uniform distribution of all physical parameters along the line of sight. The physical conditions are obtained from the complete solution of the UST equations system of statistical equilibrium without limitation of number of quantum levels. This is achieved by the introduction of the «effective» continuum and using the appropriate probabilities of elementary processes. In the UST system only the processes of thermal excitation of plasma takes into account. As initial entered data we used observed intensities $I_{m 2}$ of the Balmer series of hydrogen atom, which have belonged to the flare «core» brightening in spectrograms. The incoming data are represented by two parameters (fig.1). These are the central intensity $I_{32}$ of $H_{\alpha}$ line and the top-level number $m_{v}$ of the last observed line of the Balmer series. The additional parameter is the Doppler width $\Delta \lambda_{D} / \lambda$ of the line. The output physical parameters: $n_{H}, \mathrm{~cm}^{-3}$ is the hydrogen atoms dense, electron temperature $T_{e} K$, and the object length along the line of sight $l, \mathrm{~cm}$.

The increasing of both the $\mathrm{H}_{\alpha}$ intensity and the number of observed lines of the Balmer series really is associated with increasing the optical thickness $\tau_{23}$ of objects in the $\mathrm{H}_{\alpha}$ line. But the results of calculations indicate in two possibilities of this behavior. In small intensities $\mathrm{I}_{32}$ the increasing $\mathrm{m}_{\mathrm{v}}$ reflects the growth of plasma density $\mathrm{n}_{\mathrm{H}}$. And if the intensities are enough high, mainly changing the length of the object along the beam. The hydrogen atom density $\mathrm{n}_{\mathrm{H}}$ is the same in all the flares enough power (fig.4a). It increases mainly just for the faint objects. The behavior of both density and temperature shows the qualitative similarity of the UST system to data both that is obtained in the VAL solar atmosphere model (VAL model, Vernazza et al., 1973) and for flares (Machado \& Linsky, 1975). The threshold values of the $\mathrm{n}_{\mathrm{H}}\left(\sim 10^{17} \mathrm{~cm}^{-3}\right), \mathrm{n}_{\mathrm{e}}\left(\sim 10^{13} \mathrm{~cm}^{-3}\right)$ and $\mathrm{T}_{\mathrm{e}}(5500$ $\mathrm{K}$ ), as well as their behavior with height (with increasing $\mathrm{I}_{32}$ ) in the atmosphere are also similar. But the scale options are significantly different. The VAL model of hydrostatic balance leads to a very small range of altitudes ( $2400 \mathrm{~km})$ above photosphere. In our model, the discrepancies between observing and modeling are not arisen.

All physical plasma characteristics in this model are proved to follow by the observed intensities of spectral lines. The increase in the object both temperature, density, and the scale are increased along the beam with the increasing of the line intensities. The patterns are accordance with the occurrence features of EAS and $C A S$ arch systems in spectral lines. Changes in spectral lines with smaller arched systems found, when the observer is removed (fig. 4.6.1 and other figures below see Ostapenko, 2012). This effect should be considered while observing the same formation with two remote locations, for example, a terrestrial observer and a spacecraft. It is important to note the fact of the existence of two types of bright seats in the flare spectra (Ostapenko \& Palush, 1982). In first, this is the overlapping of of the EAS active region and the CAS arch systems of new magnetic flux (Ostapenko, 2012; fig. 2.5.3). Secondly, each $E A S$-structure has its own more vivid places (fig.2.5.1). They are projections of those places where the observer line of sight slips along arch branches of EAS systems. Actually, the observer sees the end of thin in cross-section plasma pillar or loops arcades. Here is the complete analogy with the photo-view of the shell of planetary nebulae (fig.2.5.4). Other bright areas are believed by the actually flare-«core». Lines profiles of flares are profiles of those or other brighter areas in the flare spectrograms (fig.6.1.1; fig.3.9.2). But then, all previously obtained lines profiles of flares are inadequate to use as the consumption flare patterns. Analysis of observed line profiles are carried out in the assumption of the inadequate model of horizontal static plasma layer. When we make of the photometry of flare lines, the spectrum of the comparison we must to elect as close as possible to the research place. Profiles are not released from the $E A S$-radiation. In the applicable models are used usually by authors are incorrect neither mechanisms for extension profiles nor probabilities of photons go out.

The plasma column length on the rays view depends on the arch systems length of active region. This length is also associated with flare magnificence as an area measure occupied by arch arcade in the view plane of the observer. So, the length of a plasma column in a bright place will correlated with the area (magnitude/ball) flares and the flare intensity $\left(I_{32}\right)$ in $H_{\alpha}$ line. From fig.4.6.1c it is seen that quite the same correlation occurs with the calculated values of the electron temperature $T_{e}$ and total energy flare emission $E_{\Sigma}$ into all lines and continuums. The deviations from the general course of the curve are also quite clear both for computed flare lengths along the beam and for observational flare areas. In the first case, sub-flares bright places are a total $C A S$ radiation of compact arch systems (fig.2.5.3). In the second case, for sufficiently extend flare 
arcades, the flare area is defined as the area's only flare ribbons (fig.2.5.4), which are only the plasma column radiation of the $E A S$ arch systems. The tops of the EAS loop arcades are not seen in filter images.

\section{Common Analysis Of Discrete And Continuous Radiation Of Hydrogen P;Asma}

Two-dimensional MF4A photometry of 15.07.1981 flare echelle spectra allowed detecting of the $c s B L F$ phenomenon in the spectrum (Ostapenko, 1997). Now all the flares from the sub-flares to the most powerful $W L F$ flares are linked in one single $c S(T R F-B L F-W L F)$ process of development of the current sheets in continuous radiation. To calculate the continuous emission of hydrogen is taken the same model of horizontal layer of hydrogen plasma. In the homogeneous distribution of the physical parameters along the beam through the layer, the equation of radiation transfer is:

$$
I_{r e s}=I_{s u n} * e^{-\tau}+S_{o b j} *\left(1-e^{-\tau}\right)=I_{p h o t}+I_{c s} \text {, where: } \tau=k^{*} * N^{*} * L \text {. }
$$

Here $\tau$ - optical thickness of the layer, the $L$ - length along the beam. Absorption coefficient $k$ of negative ion of hydrogen is in the form of (fig.4; Ambartsumjan et al, 1952):

$k^{-}=110^{-17}+2.510^{-17} *[(\lambda-2000) / 4000]$.

The physical characteristics in the CS condensed layer are obtained from the solution of the UST equations system for flare discrete emission (fig.4a; Ostapenko, 2012; 50 flares, table 4.6.1) across the range of their capacities. The ratio of $N_{e} / N_{n}$ and $N_{n} / N_{n}$ defines the number of ionized («e») and neutral ( $(n »)$ ) hydrogen atoms to complete their number ( $« H \gg)$. The running conditions of the $N_{e}<<N_{n}$ (or $N_{H}<<N_{n}$ ) are usually performed and they are equal only at the moment of the $N_{e}=N_{H}$ passing point. At this point, the option $N$, which is a number of negative ion of hydrogen (from the Saha formula), has a maximum value. The value of $N$ is determined by multiplying $N_{e} * N_{H}$, which is performed at the T $\sim 10000{ }^{\circ} \mathrm{K}$ (asterisks on fig.4a). As a result, the further raising of the plasma temperature $\left(\mathrm{T}>9500{ }^{\circ} \mathrm{K}\right)$, leads to a decrease in radiation intensity of the $c s W L F$. Through the full number $\left(N_{n}\right)$ of hydrogen atoms in plasma, these data help to find partial relationships in various states such as values of neutral $\left(N_{H}\right)$, ionized $\left(N_{e}\right)$ and negative ions $(N)$.

$\lg \frac{N_{e}}{N_{H}}=0.092 \sqrt{T-5000}-6 ; \quad N_{c s}=N_{n}=N_{u}+N_{e}+N ;$

The number of negative hydrogen ions $(N)$ is calculated by the formula Saha through $N_{H}, N_{e}$ and $T$.

Function $S_{o b j}$ sources in (5.4.1) expression is determined through the coefficient of absorption:

$$
S_{o b j}=1.16^{*} 10^{17} k^{-} N^{-} /[\exp (1.44 / k T)-1]
$$

The $I_{\text {res }}$ emission intensity of the $C S$, which is calculated according to the formula 5.4.1, is shown in table 10.4.1 to set parameters and more detail the CS manifestation is shown in fig.5.4.1b,c. The each picture corresponds to its value $N_{c s}$ of the density of hydrogen atoms in the layer. The $I_{\text {res }}$ radiation comes from the $C S$ layer, which is located in chromosphere on the background of the outer photosphere $\left(I_{\text {sun }}\right)$ emissions. The intensity of the $I_{\text {res }}$ presented in relative (to the $I_{\text {sun }}$ ) units and varies from $I_{\text {res }}=0$ (full absorption of $I_{\text {sun }}$ ) to the $I_{\text {res }}=1$ (The $I_{\text {sun }}$ radiation passes he layer free).

The increasing of plasma density in the layer $\left(10^{16}<N_{c s}<310^{17}\right)$ is accompanied by the scattering of solar radiation that we view as the $c s B L F$ object. The compacted layer has the temperature $T_{c s} \sim 7000-11000^{\circ} \mathrm{K}$ and the length in the line of sight $L_{c s} \sim 1-100 \mathrm{~km}$. The $c s B L F$ strip is weakened gradually with the rise of the plasma density in the layer of $310^{17}<N_{c s}<10^{18}$. It is linked with the growing of $I_{c s}$ intensity of own sources of hydrogen excitation in the layer. On reaching the plasma density in the layer of $N_{c s} \sim 10^{18} \mathrm{~cm}^{-3}$, the flare $c s B L F$ strip is fully filled by the $c s W L F$ radiation, which the observer does not see in the photosphere background. The $c s W L F$ an observer will register only at further increase of plasma density $N_{c s}>10^{18} \mathrm{~cm}^{-3}$. If our model is correct, the problem of flaring activity turns out to be solved. But then, it is appeared that the solar flare do is the current sheet $(C S)$ directly. Hence, the primary source of flare energy is magnetic field in already by definition of the $C S$ theory. The transmission mechanism of energy is the reconnection of force lines in the current sheet. The external view of flares by all power reflected the plasma density growth in the $C S$. When $N_{n}>10^{18}$, the white light flare ( $c s W L F$ ) intensity is growing much more rapidly with density $N_{n}$ in $C S$ (fig.4) and an observer will register «explosive» appearance of the white light $(c s W L F)$. All solar flares are collected in a single process of $c s(T R F-B L F-W L F)$ evolution in rise increasing of hydrogen plasma excitation (Ostapenko, 2012).

The density increasing of hydrogen atoms to the $N_{c s}>10^{14} \mathrm{~cm}^{-3}$ transforms the model layer of chromosphere formation to photosphere one. Just starting with such densities is subject to LTE in Layman lines (fig.4.3.1). In the spectrum few of hydrogen, $H \& K$ CaII, and some metal lines are only seen. The flare 
continuous radiation is absent, although the current sheet have formed and the plasma density into it is growing steadily. This is demonstrated by the emergence, after a while, the elongated wings of hydrogen and $H \& K C a I I$ lines. On reaching the plasma density $N_{c s}>510^{16} \mathrm{~cm}^{-3}$ (fig. 5.3.2), the $C S$ layer has immediately manifested itself in a spectrum by the $c s B L F$ strip. The $c s B L F$ appearance means the first excitation signs of the negative ion of hydrogen.

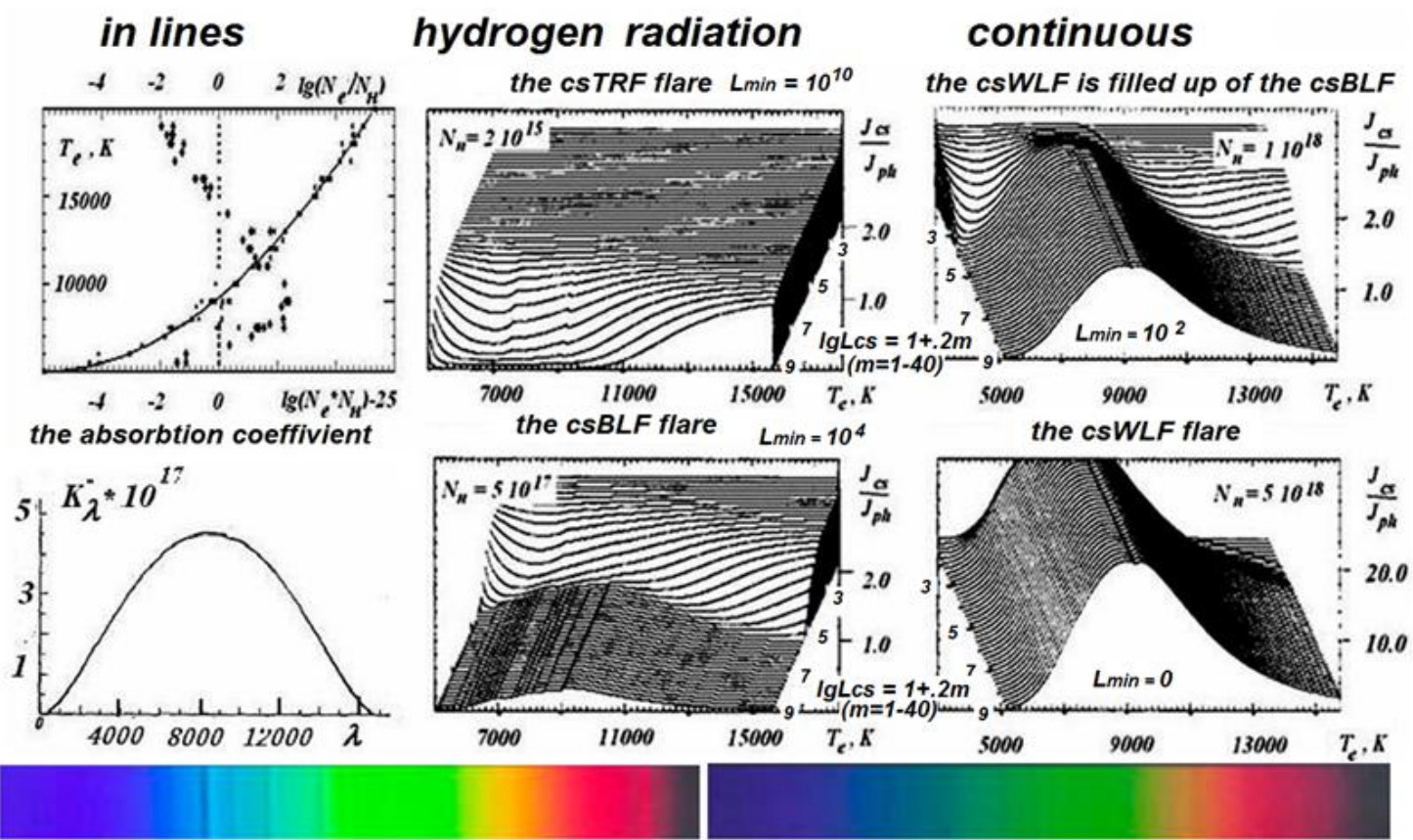

Fig.5. The solution results of (5.4.1) equation transfer of the continuous emission of hydrogen negative ion simultaneously with hydrogen emission lines. In the process of the flare $c S(T R F-B L F-W L F)$ evolution, the length $L_{c s}$ of the CS layer along the beam is reduced from $10^{10}$ to $1 \mathrm{~cm}$.

APOD-23.08.2013 - Spectra (negative hydrogen ion) of Nova Delphini \& G5 star (right)- Jurg Alean.

$\boldsymbol{a}$. The $N_{e} / N_{H}(e)+N_{H} N_{e} \quad(H)$ ratios and $N_{e} * N_{H} \quad \boldsymbol{b}$, $\boldsymbol{c}$. Views in the continuous spectrum of hydrogen (asterisk) depending on the plasma temperature. compressed chromosphere layer over the photosphere in Approximation of this data is shown in the upper the (fig.2.2.1) model for the characteristic stages of the figure squares. The bottom shows the spectral $c s(T R F-B L F-W L F)$ evolution when CS plasma density dependence of the continuous absorption $N_{n}=210^{15}, 510^{17}, 10^{18}, 510^{18} \mathrm{~cm}^{-3}$ and the set of values coefficient of hydrogen negative ion of temperature $\mathrm{T}_{\mathrm{e}}=(5-15) 10^{3} \mathrm{~K}$ and its stratum along the (Chandrasekhar, 1946). beam of the $L_{c s}=\left(10^{9} \mathrm{~cm}-10 \mathrm{~cm}\right)$.

The number of negative hydrogen ions depends on the product of the number of hydrogen atoms and the number of free electrons. And this circumstance is the stabilizing factor. A rise in temperature (degrees of ionization plasma) leads to a rise in the number of free electrons and to decrease the number of neutral hydrogen atoms (hence, and the number of negative hydrogen ions). Such temperature stabilization does not explain the weakening intensity of spectral lines in the «white» flare. It role here is played the start of plasma turbulence that follows from the theory of the internal structure of the Sun. The convective zone and hydrogen continuous emission are arisen in plasma density namely in $>10^{17} \mathrm{~cm}^{-3}$. This compatibility between our $C S$ simulation results and the results of the internal Sun's structure allowed is sure of the correctness of our calculations. The MF4A photometry also shows additional arguments in favor of the reality of this process in the current sheet when we achieve of new effects in exactly the same plasma density.

We note a number of regularities in the $c s B L F \& c s W L F$ that are appearing on the background of general flaring process. The radiation mechanism both $c s B L F \& c s W L F$ is the excitation of negative ion of hydrogen. A continuous spectrum of solar flares occur in flare $C S$ condensed plasma layers, which are formed in the chromosphere in the initial phase of flares development. The $C S$ layers are characterized by temperature $8000-10000{ }^{\circ} \mathrm{K}$, plasma density $10^{17}-10^{18} \mathrm{~cm}^{-3}$ and a line of sight length of $1 \mathrm{~cm}-100 \mathrm{~km}$. Visually in the spectrum, the compacted layer is observed in the CS-bands form of continuous either $c s W L F$ emission or $\operatorname{csBLF}$ absorption. The $C S$ strip occurs (Svestka, 1976) simultaneously with the growing of Balmer lines number to the $H_{12}$ and the lengthening wings of hydrogen and $H \& K$ CaII lines. At the same time, on the background strips of continuous radiation are appearing numerous lines of metals. In $H \& K C a I I$ lines, where the $E A S$ arch systems of active region are good prominent, there is the $C A S$ flare kernel (the flare impulsive radiation component). This is 
The Full Calculate Of Discrete And Continuous Radiation Of Hydrogen Plasmas For Solar Active..

the compact arch structure of the new magnetic flux. In $N_{c s} \sim 10^{17} \mathrm{~cm}^{-3}$ plasma densities, the $C S$ compacted layer actively scatters of photosphere radiation. In the spectrum will be the $c s B L F$. If plasma densities in the layer rising to $N_{c s}>510^{17} \mathrm{~cm}^{-3}, c s B L F$ is replaced by $c s W L F$.

\section{Conclusions}

The model calculations of the linear hydrogen plasma emission are produced by the author for the horizontal compacted stable chromosphere layer. The results confirm good traditional ideas about the physical condition of chromosphere flare plasma in the optical range due similar ground model representations. Observed line profiles and mechanisms of their broadening, including the influence of radiation transfer effects in the same model, in general, are placed in the overall scheme of these representations. The linear hydrogen emission is provided by thermal excitation mechanism (electron impacts and photosphere and own emission fields).

The absence of the profile flat tops with significant optical thicknesses is due to the influence of intensity turbulence in emission volume. The theory of the radiation transfer in homogeneous plasma leads to the appearance of the fade intensity in the profile top, although these profile peculiarities are smoothed by own movements of radiation elements (Ostapenko, 2012; fig.2.4.4). The VAL-C models, which atmosphere are in the hydrostatic equilibrium shown the height of $\sim 10^{3} \mathrm{~km}$ comparison of height of the real solar atmosphere reaches of $\sim 10^{5} \mathrm{~km}$.

Self-agreed data about the physical state of the flare plasma have been computed from solutions of equations system (UST) of the statistical equilibrium for the linear/discrete radiation. Similar separate and notagreed data about physical parameters for chromosphere, prominences, and flares are available. We for the first time have obtained the homogeneous data about the flares and immediately across the all range of their capacities. As the input data we used the observed intensities of Balmer emission lines of hydrogen atom. The problem of complete and reliable observed data is solved. The compiling and solving method of the UST system is developed without limiting the number of quantum levels. The problem of self-agreed probabilities for all the elementary processes of ionization and excitation of hydrogen atom is solved. The problem of Layman radiation field is solved to be important for the correct assessment of the number of hydrogen atoms in the ground quantum state. The terrestrial observations are not allowed to receive the data in UV-range. We used for the first time the real expression for Lyman-alpha radiation.

We have obtained the solution for 50 solar flares of varying capacity. With all faithfully to these values the physical conditions in the approach of the model, these characteristics have a distant relationship to the real physical conditions in flares. The whole question is consist in the complete inadequacy between by the actual conditions of the formation of hydrogen spectral line profiles for solar flares and by models of horizontal layer. Solar flares in optical range are 3D-formations. They are of loop systems or entire arcades with intense plasma flows. The same behavior of «physical» and geometrical characteristics of the object is understood namely in the flare arch model. It is impossible to be confident in the correctness of conclusions not only on physical conditions, but even about mechanisms of flare plasma excitation.

The field problems of Lyman-alpha radiation have been considered and resolved. The empirical expression is obtained, which has its own physical sense. These Lyman-alpha data with the results of the analysis of continuous radiation have allowed to install by the physical sense of different zones of the solar atmosphere (photosphere, transition region, chromosphere, and corona).

The transition zone is the place of formation of the solar wind. The transition zone is the place, where the mechanisms of energy transfer from the internal layers of the Sun are changing. Here energy of the radiative transfer is changed in energy by plasma jets out lot of small current sheets in the space between the granules. Convective elements are observed as the photosphere granulation. It was the granular structure that creates the conditions of formation by the current sheets in the photosphere-chromosphere transition zone of convective structure with its distinctive field of mass plasma flows. The new concept of transitional zone is agreed well with the current sheets available in the Sun and their direct observed manifestations in the photos as well as with the photometry using of solar wind manifestations.

The continuous emission of hydrogen negative ion in Sun's chromosphere plasma is calculated. The initial physical characteristics of chromosphere plasma are simultaneously found in result of solution of the UST equations system of statistical equilibrium for line emission of flare hydrogen atom in all range of their powers. The continuous emission of the $C S$ (radiation of hydrogen negative ion) is characterized for its development by the following main parameters: a) the plasma densities $N_{c s} \sim 10^{14}-10^{18} \mathrm{~cm}^{-3}$; b) temperature $T_{c s} \sim 9000^{\circ} \mathrm{K}$; c) the length along the beam $L_{c s} \sim 10^{5}-10^{1} \mathrm{~cm}$. The magnitude of the $L_{c s}$ is decreased as $N_{c s}$ is increased. Maximal attainable development stage of each flare is determined by the strengths of interacting magnetic fields. When the plasma densities in the $C S$ layer are $N_{c s}<510^{16} \mathrm{~cm}^{-3}$ (weak flares), the $C S$ is transparent (csTRF) for the continuous emission of the photosphere. Continuous $c s B L F$ emission ( «black» flares) is appeared when plasma density are $510^{16}<N_{c s}<710^{17} \mathrm{~cm}^{-3}$ (flares of middle power). In powerful flashes $\left(N_{c s}>710^{17} \mathrm{~cm}^{-3}\right)$, the $c s B L F$ is replaced by $c s W L F$ «white» emission. 
A typical «white» flare occurs by individual bright knots $\sim 5000 \mathrm{~km}$. Maximal knots brightness are $\sim 175 \%$ of the brightness of the adjacent quiet photosphere. The width of the $H_{\alpha}$ line profile is growing fast within 5 minutes and at the time of the appearance of flare in the integral light reaches $17 \AA$. By increasing in size, a flare takes the appearance of an arch system. Arch structures are continuously growing in the direction from the bottom to up. The $c s W L F$ flare is appeared, when the $H_{\alpha}$ line width $>20 \AA$. The $c s W L F$ is destructed, when the $H_{\alpha}$ line width $>40 \AA$ Á.

Calculations using line and continuous radiation of hydrogen plasma, simultaneously with observations and spectra photometry with 40-channel MF4A, clarify all basic manifestations of flares and flare-like events in solar plasma. This proven and correctness of calculations performed.

Geometric measures of CS are $10^{5}-10^{1} \mathrm{~cm}$. An emergence of a new magnetic flux in an active region, in suitable conditions, this is the time of a chromosphere $C S$ origin (in typical altitudes of $5-1010^{3} \mathrm{~km}$ above the photosphere) and the time of a flaring process origin. For the occurrence of flare is not required nor having a coronal current sheet, nor the process before-flare accumulation of energy. A flare is not a destruction process of the already existing coronal $C S$. A flare itself is the current sheet. All flare energy is provided by a $C S$ plasma flows.

A $c s(T R F-B L F-W L F)$ process is very fast (tens of seconds or minutes). If a current sheet is located vertically, its cumulative effect in optical images of limb flares is caused by the same effect of a CS own and shows simultaneously upward (CMEs) and downward (shock wave or tsunami).

\section{References}

[1]. Ambartsumjan V. A., Mustel E. R., Severny A. B., and Sobolev V. V. Theoretical Astrophysics. M., "GITTL", 1952, 635 p. (ru).

[2]. Chultem T. \& Yakovkin N. A. Stationary equations of hydrogen excitation and ionization in prominences. Solar Phys., 1974, v.34(1), p.133-150. SAO/NASA Astrophysics Data System (ADS). http://articles.adsabs.harvard.edu//full/1974So $\mathrm{Ph} . . .34 . .133 \mathrm{C} / 0000135.000 . \mathrm{html}$.

[3]. Conway M. A. Theory of Doppler line broadening and self-absorption. Contr. Dun. Obs., 1962, No.3, p.1-5.

[4]. Dupree A. K., Huber M.C.E., Noyes R. W., Parkinson W. H., Reeves E. M., and Withbroe G.J. The extreme-ultraviolet spectrum of a solar active region. Ap.J., 1973, v.182, p.321-333.

[5]. Eljashevich M. A. Atomic and Molecular Spectroscopy. M., "FM", 1962, 892 p. (ru).

[6]. Feiter de L. D. Analysis of the Balmer spectrum of solar flares, "Rech. Astron. Obs. Utrecht", 1966, v.10, p.1-79.

[7]. Golden L. B. \& Sampson D. H., Semi empirical cross-sections and rates for excitation and ionization of hydrogen by electron impact. Astrophys. J, 1971, v.163, p.405-410.

[8]. Gryzinski M. Classical theory of electronic and ionic inelastic collisions, Phys. Rev., 1959, v.115, p.374-383.

[9]. Johnson L. C. Approximations for collisional and radiative transition rates in atomic hydrogen. Astrophys. J., 1972, v.174, p.227236.

[10]. Kingston A. E. The populations of the discrete levels of atomic hydrogen in the chromosphere. Astrophys. J., 1964, v.140, p.736741.

[11]. Krivsky L., Kurochka L. On Lyman's emission of solar flares. Bull. Astron. Inst. Czechosl., 1974, v.25, p.52-61.

[12]. Kurochka L. N., Kurochka E. V., Ostapenko V. A. Radiation of chromospheres' flares in hydrogen lines. Bull. Astron. Inst. Czechosl., 1975, v.26, p.23-29.

[13]. Kurochka L. N. \& Ostapenko V. A. Physical conditions in powerful solar flares. Solnechnye Dannye (Pulkovo), 1978, No.6, p.6774; 1978, No.7, p.82-91 (ru).

[14]. Machado M. E. \& Linsky J. L. Flare models chromospheres and photospheres. Solar Phys., 1975, v.42, p.395-420.

[15]. Makarova E. A. \& Kharitonov A. V. Average data of the absolute distribution of energy in the Sun' spectrum in the field of 1800 up to $4 \mathrm{~mm}$. Solar constant. Astron. J, 1968, v.45, p.752-765.

[16]. Menzel D. H. \& Pekeris C. L. Absorption coefficients and hydrogen line intensities, M. N. R. A. S., 1935, v.96, p.77-111.

[17]. Ostapenko V. A. Probabilities of ionization and excitation of hydrogen atoms of electron impacts. Vestnik Kiev. University, Astronomy, 1974, v.16, p.42-54 (ru).

[18]. Ostapenko V. A., Rossada V. M. The spectra photometries of the limb chromosphere flare on 11 August, 1972. Solnechnye Dannye (Pulkovo), 1976, No.12, p.91-101 (ru).

[19]. Ostapenko V. A., Kurochka L. N. Physical conditions in chromosphere flares of the middle power. Vestnik Kiev. un-ty, Astronomy, 1978, No.20, p.14-29 (ru).

[20]. Ostapenko V.. \& Palush P., Spectral manifestations of the spatial structure and dynamic processes of chromospheric flares on the Sun. Bull. Astron. Inst. Czech., 1982, v.33, p.338-345.

[21]. Ostapenko V. A. \& Polupan P. N. Thermal radiation mechanism and physical "conditions" in the chromosphere $\backslash$ flares in the Sun. Problems of Cosmich. Physics, 1984, No.19, p.34-41 (ru).

[22]. Ostapenko V A. About the nature of solar flares. Vestnik SNAU, 2005, v.13, p.135-146 (ru). (SNAU - Sumy' National Agrarian University).

[23]. Ostapenko V. A. Solar flares: current sheets on the Sun, ecological power on the Earth. Kiev, "Ukraine”, 2012,185 p.

[24]. Polupan P. N. \& Yakovkin N. A. Investigations of the limb chromosphere' flare. Astron. J., 1965, v.42, p.764-774 (ru).

[25]. Presnyakov L. Atoms' ionization by electron impacrs, JETF, 1964, v.47, p.1134-1135 (ru).

[26]. Sampson D. H. \& Golden L. B. Electron-impact excitation and ionization cross-sections and rates for hydrogen, Astrophys. J, 1970, v.161, p.321-337.

[27]. Science \& Technology News since 1998 (SciTech Daily). http://scitechdaily.com/lyman-alpha-blobs-are-some-of-the-largestindividual-objects-in-the-observable-universe/

[28]. Smith H. \& Smith E. Solar Flares. (ed. A. B. Severny), M., "Mir”, 1966, 426 p.

[29]. Sobolev V. M. Investigations of excitation of hydrogen and helium in solar prominences. Izv. GAO in Pulkovo, 1958, No.158, p.12-66 (ru)

[30]. Sobolev V. M. Stationary distributions of hydrogen atoms on states in etmosphere of the Sun. Izv. GAO in Pulkovo, 1961, No.167, p.52-85 (ru). 
[31]. Svestka Z. Solar Flares. D. Reidel Co., 1976, 400 p.

[32]. Vainstein L., Presnyakov L., Sobelman I. About one model for calculate of effective cross-sections of excited atoms, JETP, 1963 ,v.45, p.2015-2021 (ru).

[33]. Vernazza J. E., Avrett E. H., and Löeser R. Structure of the solar chromosphere I. Basic computations and summary of the results. Astrophys. J., 1973, v.184, p.605-631.

[34]. Yakovkin N. A. On the mature of chromosphere' flares. Solnechnye Dannye (Pulkovo), 1963, No.8, p.62-68 (ru). 